\title{
Artificial Intelligence in Obstetrics and Gynaecology: Is This the Way Forward?
}

\author{
ELIF ILIRIA EMIN ${ }^{1}$, ECE EMIN ${ }^{2}$, APOSTOLOS PAPALOIS ${ }^{3}$, \\ FREDRIC WILLMOTT ${ }^{4}$, SONJI CLARKE ${ }^{5^{*}}$ and MICHAIL SIDERIS ${ }^{6 *}$ \\ ${ }^{1}$ Faculty of Life Sciences and Medicine, King's College London, London, U.K.; \\ ${ }^{2}$ School of Biosciences, Kingston University London, London, U.K.; \\ ${ }^{3}$ Experimental Educational and Research Centre ELPEN, Athens, Greece; \\ ${ }^{4}$ Whipps Cross University Hospital Barts Health NHS Trust, London, U.K.; \\ ${ }^{5}$ Guys and St Thomas' Hospital, King's College London, London, U.K.; \\ ${ }^{6}$ Women's Health Research Unit, Queen Mary University of London, London, U.K.
}

\begin{abstract}
An increasing trend in funding towards artificial intelligence (AI) research in medicine has re-animated huge expectations for future applications. Obstetrics and gynaecology remain highly litigious specialities, accounting for a large proportion of indemnity payments due to poor outcomes. Several challenges have to be faced in order to improve current clinical practice in both obstetrics and gynaecology. For instance, a complete understanding of fetal physiology and establishing accurately predictive antepartum and intrapartum monitoring are yet to be achieved. In gynaecology, the complexity of molecular biology results in a lack of understanding of gynaecological cancer, which also contributes to poor outcomes. In this review, we aim to describe some important applications of AI in obstetrics and gynaecology. We also discuss whether AI can lead to a deeper understanding of pathophysiological concepts in obstetrics and gynaecology, allowing delineation of some grey zones, leading to improved healthcare provision. We conclude that AI can be used as a promising tool in obstetrics and gynaecology, as an approach to resolve several longstanding challenges; AI may also be a means to augment knowledge and assist clinicians in decision-making in a variety of areas in obstetrics and gynaecology.
\end{abstract}

This article is freely accessible online.

*These Authors contributed equally to this study.

Correspondence to: Michail Sideris, Women's Health Research Unit, Queen Mary University of London, London, E1 2AB, U.K. E-mail:m.sideris@qmul.ac.uk

Key Words: Artificial intelligence, obstetrics and gynaecology, personalised medicine, gynaecological cancer, fetal monitoring, IVF, review.
Obstetrics and gynaecology are highly litigious specialities, accounting for a large proportion of indemnity payments. Between 2017 and 2018, the NHS reported the sum of $£ 4,513.2$ million in total indemnity payments, with $48 \%$ of it being for obstetric and $2 \%$ for gynaecological negligence claims. Interestingly, of the total claims, obstetrics and gynaecology accounted for only $15 \%$ (1). Aside from litigation costs, long-term socioeconomic consequences of several medical errors in obstetrics can be equally detrimental. A common adverse event in maternity care is hypoxia-induced encephalopathy, which in 2015 had an overall incidence rate of 5.14 per 1,000 live births (2). This can occasionally be attributed to misinterpretation of intrapartum fetal monitoring and it is generally agreed that $50 \%$ of such cases are preventable (3).

Several challenges and poor outcomes have also been reported in gynaecology. This includes, gynaecological oncology, where failure to diagnose malignancies early affect treatment provision and prognosis (4). Further to this, treatment stratification is currently based on the International Federation of Gynaecology and Obstetrics (FIGO) classification (5). This has several limitations, and occasionally is deemed inadequate to provide individualised treatment stratification approaches.

Assisted reproductive technologies, especially in vitro fertilisation (IVF), are other examples of areas with growing demand in women's healthcare provision (6). Embryo selection and several other related challenges need to be addressed in order to optimise success rates of IVF. Further to this, a rising trend in advanced genetic engineering and its applications in everyday IVF practice have generated the need for novel knowledge to improve precision in such interventions.

High quality, traditional research methodology leading to evidence-based care has always been the primary tool used to address and face most of the challenges related to women's healthcare provision. This includes clinical trials, 
cohort studies, evidence synthesis, and many other forms of classic research methodology reported in the literature. However, there are still many grey areas that traditional research has persistently failed to address and provide solutions for, possibly related to lack of applicability of many of the predictive models in clinical practice. Clinical trials and systematic reviews tend to be costly and time consuming, and this may be one of the greatest obstacles in conventional research methodology. This suggests the clear need for a novel era in addressing research questions through the judicious use of artificial intelligence.

\section{Artificial Intelligence: Is This the Way Forward?}

Artificial intelligence (AI) is defined as the use of complex algorithms in order for machines to reason and perform cognitive functions, including problem solving and decision making (7). AI is defined as comprising four essential core elements: machine learning (ML), natural language processing, artificial neural networks (ANNs) and computer vision (7). ML relies on subtle patterns and inference to make future predictions using complex datasets. ML has been shown to outperform logistic regression for prediction of surgical site infection by analysing multiple data consisting of laboratory values, diagnosis and treatment (8). Natural language processing is designed to understand human language, including incoherent speech (7). ANNs are inspired by biological nervous systems and comprise many computational units (i.e. 'neurones'). A feed-forward type of ANN developed by a team in Edinburgh has proven to predict mortality from acute pancreatitis more accurately than the use of a scoring tool (APACHE II and Glasgow Severity) (9). Computer vision is the understanding of images and videos, such as facial recognition technology. An example which incorporates computer vision is real-time analysis of laparoscopic videos for identifying steps of a sleeve gastroscopy, with overall reported accuracy up to $92.8 \%$ (10).

\section{Aim of this Review}

We explore how AI applications are addressing common challenges in obstetrics and gynaecology. The main objective of this narrative review is to describe some important applications of AI in obstetrics and gynaecology, discuss whether AI can lead to deeper understanding of several pathophysiological aspects of these specialities, and recommend areas in obstetrics and gynaecology where AI may be used to improve healthcare provision.

\section{AI in Obstetrics}

Cardiotocography (CTG) monitors the fetal heart and uterine contractions and functions as an indirect tool for screening for the development of hypoxia. It is currently used as the major tool in intrapartum decision making. CTG sensitivity is estimated to be around $60 \%$, and it continues to be debated whether this has improved perinatal outcomes since its introduction over 45 years ago (11). CTG interpretation is prone to human error and misrepresentation because of unavoidably high inter- and intra-observer variability. CTG interpretation, in other words, has a major element of subjectivity. There have been many studies looking at CTG data in order to predict fetal outcomes and help decision making. These aimed to demonstrate how ML can be an adjunct in fetal monitoring, and objectively help determine whether a caesarean section is necessary during intrapartum care.

In 2010, researchers converted fetal heart rate variability (HRV) into energy bands of movement and associated these bands with other activity of the foetus. Using these parameters as inputs, they created data segments (epochs) for normal and pathological cases. This form of supervised learning (ML) enabled detection of half of the pathological cases, with an acceptable false-positive rate of $7.5 \%$. The combination of HRV plus other parameters, compared to HRV and the individual parameters alone, led to superior predictions (3).

In 2014, a large CTG dataset was devised into a random forest classifier, a form of decision tree, using latent class analysis to identify normal and abnormal CTG patterns. In this study, the sensitivity and specificity of correct CTG interpretation were $72 \%$ and $78 \%$ respectively; those values were calculated based on a complex 'Aggregated Confusion Matrix model' (12). Another study in 2017, where CTG data, age of mother, $\mathrm{pH}$ of umbilical artery, Apgar scores, base excess and deficit were used to classify normal vaginal and caesarean section deliveries. This study processed these parameters using subsets of ML algorithms including random forest classification, Fisher's linear discriminant analysis and deep learning. The deep-learning pattern approach seemed to produce superior sensitivity and specificity scores, $94 \%$ and 91\% respectively, compared to the former studies (11).

\section{AI in Gynaecological Oncology}

Prognostication in gynaecological cancer is currently based on FIGO classification. A modern shift towards novel radiological or molecular biomarkers is emerging as the next step in treatment stratification. In a recent review, it was shown that several studies have explored the prognostic impact of $p 53$ or Kirstein rat sarcoma viral oncogene homolog (KRAS) gene mutations in endometrial cancer (13). A classic example of novel radiological biomarkers includes the stratification of pelvic tumours based on extramural vascular invasion. Despite those efforts, the complexity of the carcinogenesis pathway, as well as lack of understanding of several multifactorial 
mechanisms involved in cancer progression artist to creating a huge challenge that needs to be addressed in order to achieve the vision of personalised medicine. Moreover, predicting response to neoadjuvant or adjuvant treatment for individualised care provision in gynaecological oncology requires a similar or sometimes deeper understanding of the relevant molecular mechanisms involved. Therefore, AI algorithms are being developed to face this challenge.

Researchers at Imperial College London used radiomicsdetermined mathematical descriptors of primary epithelial ovarian cancer risk phenotype to provide a reliable and promising predictive value of prognosis (14). Software, TexLab 2.0, was employed to summarise 657 features relating to the size, shape, texture, wavelet, intensity and decomposition of 364 preoperative contrast-enhanced computed tomographic scans. These radiomic profiles were investigated alongside molecular profiling and gene expression, in particular copynumber alterations. High-grade serous ovarian cancer had distinct radiomic features that were frequently associated with copy-number alterations and had worse outcomes. A radiomic prognostic vector was therefore devised, which possessed significantly better prognostic power compared to cancer antigen-125 (CA-125) and was also predictive of an association with chemotherapy resistance and poor surgical outcomes.

Another team investigated various models to predict 5year survival in patients who had undergone radical hysterectomy for treatment of cervical cancer (15). Twentythree items of demographic and perioperative data, and tumour-related parameters of 102 patients were collected. The team found the best model for prediction of 5-year overall survival was using a probabilistic neural network.

Other groups have developed a clinical decision scoring system using ANN for women with cervical intraepithelial neoplasia (CIN). They utilised data of human papillomavirus (HPV) biomarkers (E6 and E7 mRNA, and p16 ${ }^{\mathrm{INK} 4 \mathrm{~A}}$ ) and colposcopic findings of 2,267 women. In comparison to cytology with or without HPV, the developed ANN had sensitivity and specificity of $93 \%$ and $99.2 \%$, respectively, for prediction of CIN2 (16), suggesting enormous potential for improving patient care.

\section{The Vision of Personalised Medicine}

Hanahan and Weinberg discussed the importance of the six fundamental hallmarks in cancer biology. This has been the basis of the current molecular oncology principles, and an essential step to pursue the vision of being able to offer individualised approach for each cancer patient (17). Personalised medicine can be defined as the use of combined knowledge about a person (e.g. genetics, medical history) to predict disease susceptibility, prognosis or treatment response (18). Prognostic and predictive biomarkers can guide patient management and forecast outcomes. Mutations of breast cancer gene 1 and 2 (BRCA1 and BRCA2) are a successful example of parameters for provision of personalized treatment based on individual predictors or prognosis and response to treatment (19). KRAS mutations in endometrial cancer and WNT signalling in ovarian, endometrial and cervical cancer are also potential future targets based on which prognosis of cancer progression might be predicted on an individual basis and initial treatment options therefore be stratified accordingly $(13,20)$.

Modern medicine has shifted from developing treatments after the fact, to preventing, personalising and delivering precision care. This requires vast amounts of data to increase available knowledge on disease processes. Examples of processable data include proteomic, genomic and transcriptomic biomarkers, and baseline characteristics of patients. AI has been declared as the primary tool to synthesise data on complex cancer oncology and achieve the vision of personalised medicine.

\section{AI in IVF}

The selection of a viable embryo remains a principle challenge of IVF. This is essential in order to predict outcomes which could lead to shorter time to pregnancy and result in a healthy, live-birth. In 1997, Kaufmann et al. developed an ANN with a predictive power of 59\% using only four inputs (age, number of eggs recovered, number of embryos transferred and whether there was embryo freezing) in order to predict the likelihood of successful IVF (21). Obstacles to greater sensitivity in this area include the many unknown factors which lead to a successful outcome for IVF which are needed to 'teach' for the purpose of ML (22). It is for this reason that large datasets, such as computer vision, are being used to construct an ANN in an attempt to increase predictive power.

Time-lapse imaging of embryos and image data have been used to obtain large datasets. Several studies were presented at the 2018 Annual Congress of the American Society for Reproductive Medicine and the European Society for Human Reproduction and Embryology. More specifically, one of those studies achieved $83 \%$ overall accuracy in predicting live birth by looking at 386 time-lapse images of single blastocyst transfers (23). Another study reviewed 50,392 images from 10,148 embryos and managed to obtain $97.53 \%$ accuracy in discriminating between a poor and good blastocyst (24). Others used pre-treatment characteristics of known cycles to predict first cycle success, which had an accuracy of $81 \%$ (25).

\section{Limitations}

We acknowledge a series of limitations in this narrative review. Firstly, despite the fact that a formal key word 
strategy was followed, this article has discussed only those studies which were considered to follow a robust research methodology and deemed essential for the understanding of the field. Secondly, the vast majority of the included studies related to data in an experimental phase and are yet to be applied clinically; some studies had small sample numbers and others oversampled data. In particular, with IVF studies there was a lack of explanation of how the training of machinery took place and the specific AI architecture used. There are also the limitations of AI itself, such as systematic biases and improper labelling of data, leading to skewed results. Finally, AI success really depends on large datasets, thus the speed of discovery is dependent on the speed of data availability.

\section{Conclusion}

AI is a hot topic and its application in several specialities has been associated with great expectations; an increasing trend in funding AI research reflects this. AI seems to be a promising tool in obstetrics and gynaecology for resolving several longstanding challenges. Our review concludes that AI can augment knowledge and assist clinicians in decision making in a variety of areas in obstetrics and gynaecology. CTG interpretation and fetal physiology can potentially be facilitated by AI, thereby limiting adverse events in obstetrics. In the area of gynaecology, AI can delineate the complexity of the molecular biology of gynaecological cancer and consequently serve the vision of personalised medicine.

\section{Conflicts of Interest}

None declared.

\section{Authors' Contributions}

EIE drafted the article, performed literature search and conceived methodology with MS. EE performed the literature review and assisted in drafting parts of the article. AP and SC edited parts of the article and offered senior input. MS and SC edited the final version of article; SC performed extensive editing of the primary draft. MS and SC are equal contributors. FW also offered input in the first draft. All Authors have approved the final version of the article.

\section{Funding Statement}

No funding was received for this study.

\section{References}

1 NHS, Annual report and accounts 2017/18, N.L. Authority, Editor. 2018. Available at: https://resolution.nhs.uk/wpcontent/uploads/2018/08/NHS-Resolution-Annual-Report-20172018.pdf (Last accessed July 5, 2019).
2 Gale C, Statnikov Y, Jawad S, Uthaya SN, Modi N and Brain Injuries Expert Working Group: Neonatal brain injuries in England: Population-based incidence derived from routinely recorded clinical data held in the national neonatal research database. Arch Dis Child Fetal Neonatal Ed 103(4): F301-F306, 2018. PMID: 29180541. DOI: 10.1136/archdischild-2017-313707

3 Warrick PA, Hamilton EF, Precup D and Kearney RE: Classification of normal and hypoxic fetuses from systems modeling of intrapartum cardiotocography. IEEE Trans Biomed Eng 57(4): 771-779, 2010. PMID: 20659819. DOI: 10.1109/ TBME.2009.2035818

4 Williams P, Murchie P and Bond C: Patient and primary care delays in the diagnostic pathway of gynaecological cancers: A systematic review of influencing factors. $\mathrm{Br} \mathrm{J}$ Gen Pract 69(679): e106-e111, 2019. PMID: 30642909. DOI: 10.3399/bjgp 19X700781

5 Amant F, Mirza MR, Koskas M and Creutzberg CL: Cancer of the corpus uteri. Int J Gynaecol Obstet 143(Suppl 2): 37-50, 2018. PMID: 30306580. DOI: 10.1002/ijgo.12612

6 NHS, IVF, 2018. Available from: https://www.nhs.uk/ conditions/ivf/ (Last accessed July 5, 2019).

7 Hashimoto DA, Rosman G, Rus D and Meireles OR: Artificial intelligence in surgery: Promises and perils. Ann Surg 268(1): 70-76, 2018. PMID: 29389679. DOI: 10.1097/SLA.0000000 000002693

8 Soguero-Ruiz C, Fei WM, Jenssen R, Augestad KM, Alvarez JL, Jimenez IM, Lindsetmo RO and Skrovseth SO: Data-driven temporal prediction of surgical site infection. AMIA Annu Symp Proc 2015: 1164-1173, 2015. PMID: 26958256.

9 Nadkarni PM, Ohno-Machado L and Chapman WW: Natural language processing: An introduction. J Am Med Inform Assoc 18(5): 544-551, 2011. PMID: 21846786. DOI: 10.1136/amiajnl2011-000464

10 Volkov M, Hashimoto DA, Rosman G, Meireles OR and Rus D: Machine learning and coresets for automated real-time video segmentation of laparoscopic and robot-assisted surgery. IEEE Int Conf Robotics Automat, Singapore, pp. 754-759, 2017. DOI: 10.1109/ICRA.2017.7989093

11 Fergus P, Hussain A, Al-Jumeily D, Huang DS and Bouguila N: Classification of caesarean section and normal vaginal deliveries using fetal heart rate signals and advanced machine learning algorithms. Biomed Eng Online 16(1): 89, 2017. PMID: 28679415. DOI: $10.1186 / \mathrm{s} 12938-017-0378-\mathrm{z}$

12 Spilka J GG, Karvelis P and Chudacek V: Discriminating normal from 'abnormal' pregnancy cases using an automated FHR evaluation method. In: Likas A, Blekas K and Kalles D (eds): Artificial Intelligence: Methods and Applications. SETN 2014. Lecture Notes in Computer Science, vol. 8445. Springer, Cham, Switzerland.

13 Sideris M, Emin EI, Abdullah Z, Hanrahan J, Stefatou KM, Sevas V, Emin E, Hollingworth T, Odejinmi F, Papagrigoriadis S, Vimplis S and Willmott F: The role of kras in endometrial cancer: A mini-review. Anticancer Res 39(2): 533-539, 2019. PMID: 30711927. DOI: 10.21873/anticanres.13145

14 Zhou M, Scott J, Chaudhury B, Hall L, Goldgof D, Yeom KW, Iv M, Ou Y, Kalpathy-Cramer J, Napel S, Gillies R, Gevaert O and Gatenby R: Radiomics in brain tumor: Image assessment, quantitative feature descriptors, and machine-learning approaches. Am J Neuroradiol 39(2): 208-216, 2018. PMID: 28982791. DOI: 10.3174/ajnr.A5391 
15 Obrzut B, Kusy M, Semczuk A, Obrzut M and Kluska J: Prediction of 5-year overall survival in cervical cancer patients treated with radical hysterectomy using computational intelligence methods. BMC Cancer 17(1): 840, 2017. PMID: 29233120. DOI: 10.1186/s12885-017-3806-3

16 Kyrgiou M, Pouliakis A, Panayiotides JG, Margari N, Bountris P, Valasoulis G, Paraskevaidi M, Bilirakis E, Nasioutziki M, Loufopoulos A, Haritou M, Koutsouris DD, Karakitsos P and Paraskevaidis E: Personalised management of women with cervical abnormalities using a clinical decision support scoring system. Gynecol Oncol 141(1): 29-35, 2016. PMID: 27016226. DOI: $10.1016 /$ j.ygyno.2015.12.032

17 Hanahan D and Weinberg RA: Hallmarks of cancer: The next generation. Cell 144(5): 646-674, 2011. PMID: 21376230. DOI: 10.1016/j.cell.2011.02.013

18 Redekop WK and Mladsi D: The faces of personalized medicine: A framework for understanding its meaning and scope. Value Health 16(6 Suppl): S4-9, 2013. PMID: 24034312. DOI: 10.1016/j.jval.2013.06.005

19 Cho SH, Jeon J and Kim SI: Personalized medicine in breast cancer: A systematic review. J Breast Cancer 15(3): 265-272, 2012. PMID: 23091538. DOI: 10.4048/jbc.2012.15.3.265

20 Ford CE, Henry C, Llamosas E, Djordjevic A and Hacker N: Wnt signalling in gynaecological cancers: A future target for personalised medicine? Gynecol Oncol 140(2): 345-351, 2016. PMID: 26432042. DOI: 10.1016/j.ygyno.2015.09.085

21 Kaufmann SJ, Eastaugh JL, Snowden S, Smye SW and Sharma $\mathrm{V}$ : The application of neural networks in predicting the outcome of in vitro fertilization. Hum Reprod 12(7): 1454-1457, 1997. PMID: 9262277.
22 Curchoe CL and Bormann CL: Artificial intelligence and machine learning for human reproduction and embryology presented at ASRM and ESHRE 2018. J Assist Reprod Genet 36(4): 591-600, 2019. PMID: 30690654. DOI: 10.1007/s 10815-019-01408-x

23 Zaninovic N Rocha CJ, Zhan Q, Toschi M, Malmsten J, Nogueira M, Meseguer M, Rosenwaks Z and Hickman C: Application of artificial intelligence technology to increase the efficacy of embryo selection and prediction of live birth using human blastocysts cultured in a time-lapse incubator. Feril Steril 110(4): e372-e373, 2018. DOI: 10.1016/j.fertnstert. 2018.07.1039

24 Zaninovic N, Khosravi P, Hajirasouliha I, Malmsten JE, Kazemi E, Zhan Q, Toschi M, Elemento O and Rosenwaks Z: Assessing human blastocyst quality using artificial intelligence (AI) convolutional neural network (CNN). Fert Steril 110(4): e89, 2018. DOI: 10.1016/j.fertnstert.2018.07.267

25 Correa N, Brazal S, García D, Brassesco M and Vassena R: Development and validation of an artificial intelligence based algorithm for the selection of an optimal stimulation protocols in IVF patients. Hum Reprod 33(suppl_1): i88, 2018. DOI: 10.1093/humrep/33.supplement_1.1
Received May 29, 2019

Revised July 3, 2019

Accepted July 8, 2019 